\title{
The Effects of a Warmed Steam Inhalation on Patients with Chronic Obstructive Pulmonary Disease: A Cross-Sectional, Controlled Study
}

\author{
Kawagoshi $\mathbf{A}^{1 *}$, Shibata $\mathrm{K}^{1}$, Sugawara $\mathrm{K}^{1}$, Takahashi $\mathbf{H}^{1}$, Saita $\mathbf{Y}^{2}$, Oda $\mathbf{H}^{2}$ and Shioya $\mathrm{T}^{3}$
}

${ }^{1}$ Department of Rehabilitation, Akita City Hospital, Akita, Japan

${ }^{2}$ Development Research, Personal Health Care Products Research, Kao Corporation, Tokyo, Japan

${ }^{3}$ Department of Physical Therapy, School of Health Sciences, Akita University, Akita, Japan

\begin{abstract}
Background: We investigated the influence of warmed steam inhalation on the respiratory dynamics and psychological anxiety in patients with chronic obstructive pulmonary disease (COPD).

Methods: This was a cross-sectional and controlled study of 16 patients with stable COPD (age: $72 \pm 6$ years old, $\% \mathrm{FEV}_{1}: 61.4 \pm 28.2 \%$ ). The patients wore a steam mask in a resting position for $15 \mathrm{~min}$. Two weeks later, they wore a mask that generated no warmed steam (non-steam mask) under the same conditions. We evaluated the patients' respiratory function, percutaneous oxygen saturation, heart rate (HR), and state anxiety based on the State-Trait Anxiety Inventory (STAI) before and after wearing the masks, and the respiratory rate was measured during the mask wearing.

Results: The patients' HR and STAI state anxiety were significantly decreased after they wore the steam mask. The respiratory rate was significantly decreased during the steam mask use compared to that during the non-steam mask use. A significant positive correlation was noted between the patient's breathing difficulty and change in the STAI score after the steam mask use $(r s=0.503, p<0.05)$.

Conclusions: Although the warmed steam inhalation masks did not improve the COPD patients' respiratory function, it might reduce COPD patients' anxiety. A randomized study with a greater number of patients is desired to test our findings.
\end{abstract}

Keywords: COPD; Warmed steam inhalation; State anxiety

Abbreviations: COPD: Chronic Obstructive Pulmonary Disease; GOLD: Global Initiative For Chronic Obstructive Lung Disease; FEV Forced Expiratory Volume In One Second; FVC: Forced Vital Capacity; HR: Heart Rate; STAI: State-Trait Anxiety Inventory; QOL: Quality Of Life; MRC: Medical Research Council; HOT: Home Oxygen Therapy; $\mathrm{SpO}_{2}$ : Percutaneous Oxygen Saturation

\section{Background}

Significant associations have been reported between decreases in the environmental temperature and humidity with the seasonal variations of symptom exacerbation in chronic obstructive pulmonary disease (COPD) patients whose main symptom is breathing difficulty [1] and another study demonstrated that the long-term inhalation of $37^{\circ} \mathrm{C}$ humidified air both significantly decreased the number of days and frequency of exacerbation and significantly improved the quality of life and pulmonary function in patients with chronic airway disease [2]. Regarding the mechanisms underlying these phenomena, bronchoconstriction [3] and reductions of the pulmonary function [4] and immunity against respiratory infection [5] induced by exposure to a low temperature have been reported, whereas the inhalation of warmed and humidified air improved mucociliary clearance and decreased air trapping in the bronchi [2].

Many COPD patients are in a depressive state with anxiety symptoms due to their breathing difficulty, and their daily life activities and physical functions may thus decline, contributing to their breathing difficulty and aggravate their anxiety symptoms in a vicious cycle [6]. Neural mechanisms that induce unpleasant emotions such as anxiety and fear are also involved in breathing difficulty, and the involvement of the amygdala has been reported $[7,8]$ but there has been no study of the influence of the inhalation of humidified and warmed air on an individual's psychological state or about the association with the respiratory condition in COPD patients. We conducted the present study to investigate the influence of sensory input during the inhalation of warmed and humidified air on the patient's psychological state (such as anxiety) and on the respiratory dynamics in COPD patients. Our findings help explore the possibility of the clinical application of this technique.

\section{Patients and Methods}

\section{Patients}

The patients were 16 male COPD patients being treated at the outpatient rehabilitation clinic of Akita City Hospital. They were age 73 \pm 6 years old, and their forced expiratory volume for 1 second expressed as a percentage of the forced vital capacity (\%FEV1) was $59.9 \pm 25.4 \%$ (Table 1). The Global Initiative for Chronic Obstructive Lung Disease (GOLD) disease classification [9] of COPD was I-IV, ranging from mild to severe (stage I, $n=3$; stage II, $n=5$; stage III, $n=6$; and stage IV, $n=2$ ), and the classification using an index of breathing difficulty, the Medical Research Council (MRC) dyspnea scale [10] was $0, n=2 ; I, n=9 ; I I, n=4$; III, $\mathrm{n}=0$; and IV, $\mathrm{n}=1$. The patients had no history of admission due to

*Corresponding author: Atsuyoshi Kawagoshi, Department of Rehabilitation, Akita City Hospital, Akita, Japan, Tel: +81-18-823-4171; Fax: +81-18-866-7026; E-mail: acr00009@akita-city-hospital.jp

Received September 03, 2018; Accepted September 10, 2018; Published September 16, 2018

Citation: Kawagoshi A, Shibata K, Sugawara K, Takahashi H, Saita Y, et al. (2018) The Effects of a Warmed Steam Inhalation on Patients with Chronic Obstructive Pulmonary Disease: A Cross-Sectional, Controlled Study. J Pulm Respir Med 8: 471. doi: 10.4172/2161-105X.1000471

Copyright: (2018 Kawagoshi A, et al. This is an open-access article distributed under the terms of the Creative Commons Attribution License, which permits unrestricted use, distribution, and reproduction in any medium, provided the original author and source are credited. 


\begin{tabular}{|c|c|}
\hline Variables & Mean \pm SD \\
\hline Age (year) & $73 \pm 6$ \\
\hline BMI $\left(\mathrm{kg} / \mathrm{m}^{2}\right)$ & $23.7 \pm 3.8$ \\
\hline GOLD $(\mathrm{I} / \mathrm{II} / \mathrm{III} / \mathrm{IV})$ & $3 / 5 / 6 / 2$ \\
\hline $\mathrm{mMRC}(0 / 1 / 2 / 3 / 4)$ & $2 / 9 / 4 / 0 / 1$ \\
\hline$\% F V C(\%)$ & $87.8 \pm 16.4$ \\
\hline FEV ${ }_{1}(\mathrm{~L})$ & $1.4 \pm 0.7$ \\
\hline FEV $_{1} / \mathrm{FVC}(\%)$ & $48.6 \pm 18.9$ \\
\hline $\mathrm{FEV}_{1} \%$ pred $(\%)$ & $59.9 \pm 25.4$ \\
\hline
\end{tabular}

SD: Standard Deviation; BMI: Body Mass Index; GOLD: Global initiative for Chronic Obstructive Lung Disease; mMRC: Modified Medical Research Council; FVC: Forced Vital Capacity: FEV 1 : Forced Expiratory Volume in one second.

Table 1: Baseline characteristics of subjects.

acute exacerbation within the prior 4 weeks, and at least 1 month had passed after the initiation of drug therapy. No patient indicated for home oxygen therapy (HOT) was included.

This study was performed after approval was given by the Akita City Hospital Ethics Committee and the study content was fully explained to the patients; written consent to participate in the study was obtained from all patients (approval no.: 10 in 2015).

\section{Methods}

This was a cross-sectional, blinded evaluation (for examiners), and controlled study. The patient rested in a supine position for $10 \mathrm{~min}$ and then wore a warmed steam inhalation mask (steam mask) for 15 min. The patient's respiratory function, percutaneous oxygen saturation $\left(\mathrm{SpO}_{2}\right)$, heart rate $(\mathrm{HR})$, and anxiety were evaluated before and after the mask wearing, and the respiratory rate was measured during the mask wearing. After a 2-4 week interval, as a control measure each patient wore a mask generating no steam (non-steam mask) under the same conditions as those used for the warmed steam inhalation mask, and the same procedure has done the blinded evaluation of the parameters to avoid the measurement bias.

Steam mask and non-steam mask (Kao Corporation, Tokyo, Japan): For the steam mask, a pocket was prepared in a threedimensionally (3D) shaped mask, and a steam- and heat-generating sheet was inserted (Figure 1). The oxidation reaction of iron powder was used as the steam- and heat-generating mechanism. For the sheet, a moisture-permeable material was adopted for the skin side, and the heat-generating components (mainly, iron, activated carbon, water, and salt) were encapsulated. Heat and steam were generated by the reaction of these components with oxygen in the air, and the steam was supplied into the 3D-shaped mask through the moisture-permeable material. The amount of steam generated in the mask was approx. $600 \mathrm{mg}$ per $10 \mathrm{~min}$, and the skin temperature in the mask was approx. $38^{\circ} \mathrm{C}-40^{\circ} \mathrm{C}$ persisting for about $15 \mathrm{~min}$. Burns are avoided at this temperature and duration [11] and the mask was designed so that the wearer would feel the temperature and steam as comfortable. As a control, the non-steam mask was similarly prepared without the generation of warmed steam to conceal which the steam or not for examiners.

Tests and measurement methods: For the measurement of respiratory function, a compact electronic spirometer (Chestgraph-HI 701, Chest MI, Tokyo) was used. We determined each patient's forced vital capacity (FVC), forced expiratory volume in one sec $\left(\mathrm{FEV}_{1}\right)$, $\mathrm{FEV}_{1} / \mathrm{FVC}$ ratio, and the $\%$ predicted $\mathrm{FEV}_{1}\left(\mathrm{FEV}_{1} \%\right.$ pred $)$. For these

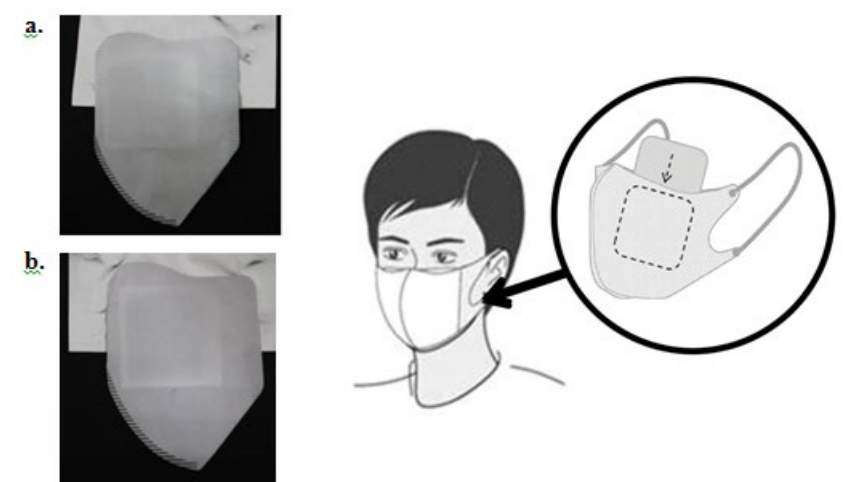

Figure 1: (a) Steam mask and (b) non-steam mask; Kao Corporation, Tokyo, Japan.

measurements, the patient sat on a chair, wore a nose clip, and held a mouthpiece between his teeth. Each measurement was repeated at least 3 times for the patient's acclimation to the apparatus, and we used the maximum FVC value reached by the patient [12]. The $\mathrm{SpO}_{2}$ and $\mathrm{HR}$ were measured with the use of a Pulsox-M24 pulse oximeter (Teijin, Tokyo), and we determined the values after the patient rested for $10 \mathrm{~min}$ in a supine position before wearing either mask and immediately after removing the mask.

The patients' respiratory rates were measured using an unconstrained respiratory meter, the TS03 (Techno Science, Numazu, Japan). For the data logger, we used a midi-Logger GL820 (Graphtec, Yokohama, Japan) (Figure 2). Each respiratory rate was measured for $10 \mathrm{~min}$ (from 5 to 15 min after the patient put on either mask). Anxiety was evaluated before and after the wearing of a mask by the Japanese version of the self-rating state anxiety scale of the State-Trait Anxiety Inventory (STAI) [13].

\section{Statistical analysis}

The paired t-test was used for comparisons of items between measurements taken before and after the wearing of each mask, and for the comparison of the respiratory rate between the two types of masks. For the identification of the correlations of the change in STAI after wearing the mask with the patients' GOLD and MRC classifications, we used Spearman's rank correlation coefficient.

\section{Results}

\section{Comparison between before and after wearing the mask}

As shown in Table 2, no significant difference was noted in the respiratory function (FVC, $\mathrm{FEV}_{1}, \mathrm{FEV}_{1} / \mathrm{FVC}$, or $\mathrm{FEV}_{1} \%$ pred) or $\mathrm{SpO}_{2}$ between before and after wearing either type of mask. The patients $\mathrm{HR}$ values were significantly lower at before steam mask compared to after steam mask $(67 \pm 11 \mathrm{bpm} v s .63 \pm 10 \mathrm{bpm}, \mathrm{p}<0.05)$. The patients' respiratory rates were also significantly lower during the steam mask wearing compared to the non-steam mask (steam mask: $133 \pm 47$ times/10 min, non-steam mask: $156 \pm 32$ times/10 min, $\mathrm{p}<0.05)$. For both masks, the STAI state anxiety scores were significantly lower after the mask wearing compared to before (steam mask: $34.9 \pm 8.8$ points $v s$. $29.3 \pm 10.7$ points, $\mathrm{p}<0.05$; non-steam mask: $34.8 \pm 8.6$ points $v s .32 .3 \pm$ 8.5 points, $\mathrm{p}<0.05)$.

\section{Correlation of change in STAI with GOLD and MRC}

A significant positive correlation was revealed between the change in the STAI score after the patients wore the steam mask and their MRC dyspnea scale score $(\mathrm{rs}=0.519, \mathrm{p}<0.05)$. No significant correlation was noted in any other index (Figure 3 ). 


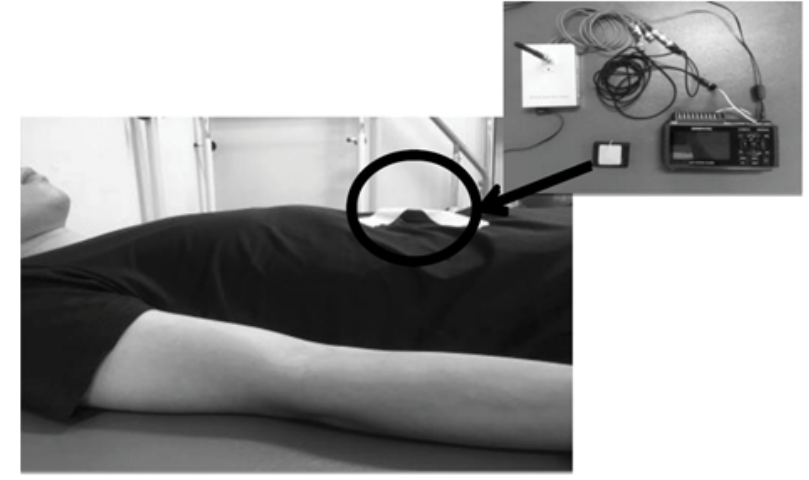

Figure 2: View of respiratory rate measurement. Respiratory meter: unconstrained respiratory meter TS03 (Techno Science Co., Ltd.). Data logger: midi LOGGER GL820, Graphtec Corporation.

\begin{tabular}{|c|c|c|c|c|}
\hline \multirow{2}{*}{ Variables } & \multicolumn{2}{|c|}{ Steam mask } & \multicolumn{2}{c|}{ Non-Steam mask } \\
\cline { 2 - 5 } & Before & After & Before & After \\
\hline$\%$ FVC $(\%)$ & $89.3 \pm 18.1$ & $86.8 \pm 20.0$ & $89.6 \pm 17.8$ & $99.5 \pm 18.7$ \\
\hline FEV $_{1}(\mathrm{~L})$ & $1.5 \pm 0.7$ & $1.5 \pm 0.7$ & $1.4 \pm 0.7$ & $1.6 \pm 0.8$ \\
\hline FEV $_{1} /$ FVC (\%) & $49.4 \pm 21.3$ & $50.9 \pm 18.3$ & $49.3 \pm 21.7$ & $49.3 \pm 19.4$ \\
\hline FEV $_{1} \%$ pred (\%) & $61.4 \pm 28.2$ & $61.1 \pm 26.5$ & $60.8 \pm 28.2$ & $68.7 \pm 30.9$ \\
\hline SpO $_{2}(\%)$ & $95.5 \pm 2.1$ & $95.2 \pm 2.7$ & $95.4 \pm 1.7$ & $95.2 \pm 2.0$ \\
\hline $\mathrm{HR}_{(b p m)}$ & $67.1 \pm 11.1$ & $63.6 \pm 9.8^{*}$ & $65.5 \pm 10.6$ & $64.5 \pm 9.6$ \\
\hline STAI (points) & $34.9 \pm 8.8$ & $29.3 \pm 10.7^{\star}$ & $34.8 \pm 8.6$ & $32.3 \pm 8.5^{\star}$ \\
\hline
\end{tabular}

Respiratory rates $133 \pm 47^{\$}$ (times $/ 10 \mathrm{~min}$ ) and $156 \pm 32$ (times/10 $\mathrm{min}$ )

${ }^{*}$ Comparison with pre, $\$$ Comparison with Non-steam mask $\mathrm{p}<0.05$; paired t-test FVC: Forced Vital Capacity: FEV $_{1}$ : Forced Expiratory Volume in one second; $\mathrm{SpO}_{2}$ : Percutaneous Oxygen Saturation; HR: Heart Rate; STAI: State-Trait Anxiety Inventory

Table 2: Comparison between before and after single wearing the mask.

\section{Discussion}

This was a pilot study to investigate the immediate influence of warmed and humidified air inhalation via a steam mask on the psychological state and respiratory dynamics of COPD patients. We also explored the possibility of clinical application of the steam mask based on the association between the patients' breathing difficulty and psychological state.

The indices of the respiratory function or $\mathrm{SpO}_{2}$ were not significantly changed by the steam mask usage, but the patients' pulse values were significantly decreased after they wore the steam mask- in contrast to the absence of a significant change after they wore the non-steam mask. In addition, the patients' respiratory rates during the steam mask usage were significantly lower than those during the non-steam mask use. Regarding the respiratory function, Harold et al. reported that a long-term inhalation of $37^{\circ} \mathrm{C}$ humidified air improved the respiratory function of patients with chronic airway disease (such as $\mathrm{FEV}_{1} / \mathrm{FVC}$ ) after 3 and 12 months of daily humidification therapy [2] but in the present study we did not observe any immediate influence of the warmed/humidified air on the patients' respiratory function, because the duration of mask wearing was short. We also detected no significant change in the patients' respiratory function or $\mathrm{SpO}_{2}$, suggesting that steam inhalation has only a small influence on the pathology.

No adverse event such as burns by steam heat occurred in our patient series, suggesting that the mask can be safely applied to COPD patients and adults with other breathing difficulties. The significant
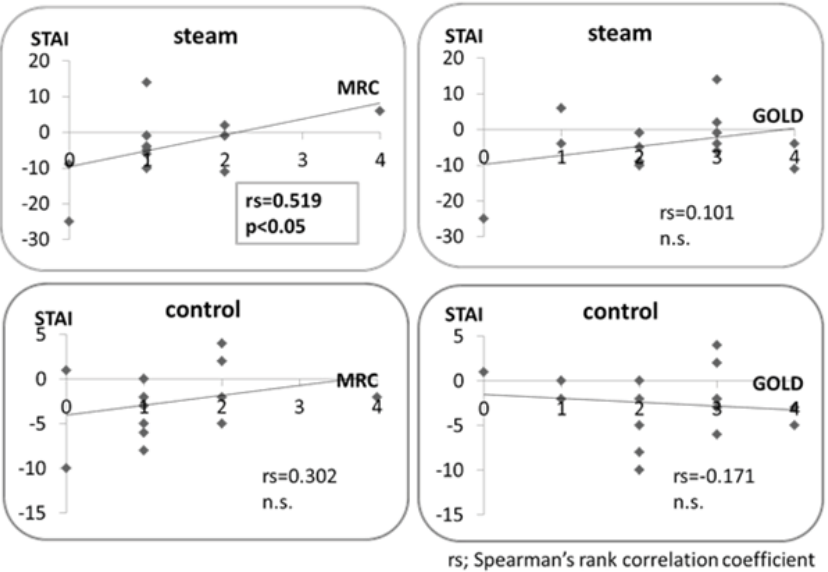

Figure 3: Correlations of change in STAI with MRC and GOLD (X-axis: the grade of MRC or GOLD; Y-axis: the changes in the STAI score after the patients wore the steam mask)

reduction of our patients' pulse values after they wore the steam mask and the significantly lower respiratory rate during the steam mask usage compared to the non-steam mask may have been parasympathetic nerve-dominant reactions, suggesting that the warmed and humidified air inhalation had a relaxation effect. As we noted in the Introduction, breathing difficulty involves brain mechanisms that are thought to induce unpleasant emotions such as anxiety and fear, and involvement of the amygdala has been reported $[7,8]$. The amygdala receives afferent information from the internal and external environments, makes alternative emotional judgements (such as pleasant or unpleasant emotions), and outputs an autonomic response at the same time [8]. In the present study, the patients' STAI anxiety scores decreased significantly after they wore the steam mask, suggesting that the warm and humid stimulation acted on the patients' emotions and resulted in a reduction of anxiety and parasympathetic nerve-dominant changes in the physiological indices.

Regarding the relationships between the change in the STAI state anxiety score and the patients' disease stage and breathing difficulty, we observed a significant positive correlation between the steam mask-induced changes in STAI and MRC scores, suggesting that the steam mask-induced reduction of anxiety is large in patients with mild breathing difficulty. Since depression and anxiety symptoms aggravate breathing difficulty in a vicious cycle in COPD patients [6] a reduction of depression and anxiety symptoms by wearing a warm steam mask beginning at the stage in which breathing difficulty is still mild may prevent the aggravation of breathing difficulty.

Regarding study limitations, since this was a pilot study involving a small number of participants, blinding of the patients was difficult and only the examiners were blinded. Moreover, the order of the tests was fixed in the study design: the steam mask was applied first, and then the non-steam mask was used. Although a 2- 4 week washout period was set, the possibility of a psychological carry-over effect cannot be ruled out. In addition to a greater number of patients, it is desirable to design a study in consideration of confounding factors due to the carry-over effect, such as a cross-over study.

\section{Conclusion}

The influence of the inhalation of warmed and humidified air through a steam mask on the respiratory dynamics and anxiety of 
COPD patients was investigated. Our patients' respiratory function and the $\mathrm{SpO}_{2}$ did not change significantly, but their pulse and respiratory rates decreased significantly with the steam mask usage, as did their state anxiety scores. In addition, a significant positive correlation was noted between the grade of breathing difficulty and the change in the state anxiety score. Although the warmed steam inhalation masks did not provide an improvement of respiratory function, anxiety may be reduced by wearing the steam mask from the stage at which breathing difficulty is still mild, and a relaxation effect can be expected. A randomized study with a sufficient number of patients could be useful as a further confirmatory study.

\section{Acknowledgements}

The authors thank the staff of the Development Research Department at the Kao Corporation (Atsushi Suzuki and Michihito Igaki) for assisting in the process of this study.

\section{References}

1. Tseng CM, Chen YT, Ou SM, Hsiao TH, Li SY, et al. (2013) The effect of cold temperature on increased exacerbation of chronic obstructive pulmonary disease: A nationwide study. PLoS One 8: e57066.

2. Rea H, McAuley S, Jayaram L, Garrett J, Hockey H, et al. (2010) The clinical utility of long-term humidification therapy in chronic airway disease. Resp Med 104: 525-533.

3. Koskela HO, Koskela AK, Tukiaineu HO (1996) Bronchoconstriction due to cold weather in COPD. The roles of direct airway effects and cutaneous reflex mechanisms. Chest 110: 632-636

4. Donaldson GC, Seemungal T, Jeffries DJ, Wedzicha JA (1999) Effect of temperature on lung function and symptoms in chronic obstructive pulmonary disease. Eur Respir J 13: 844-849.
5. The Eurowinter Group (1997) Cold exposure and winter mortality from ischaemic heart disease, cerebrovascular disease, respiratory disease, and causes in warm and cold regions of Europe. Lancet 349: 1341-1346.

6. Yohannes AM, Willgoss TG, Baldwin RC, Connolly MJ (2010) Depression and anxiety in chronic heart failure and chronic obstructive pulmonary disease: Prevalence, relevance, clinical implications and management principles. Int J Geriatr Psychiatry 25: 1209-1221.

7. Arita $H(2006)$ Mechanism of shortness of breath: General. COPD Frontier 15 $50-55$.

8. Honma I (2009) Psychosomatic adjustment and respiration. J Human Art Sci 5: 1-7.

9. NHLB/WHO workshop report (2013) Global initiative for chronic obstructive lung disease: Global strategy for the diagnosis, management and prevention of chronic obstructive pulmonary disease- executive summary. National Heart, Lung and Blood Institute, Bethesda.

10. Celli BR, MacNee W, Agusti A, Anzueto A, Berg B, et al. (2004) Standards for the diagnosis and treatment of patients with COPD: A summary of the ATS/ERS position paper. Eur Respir J 23: 932-946.

11. Moritz AR, Henriques FC (1947) Studies of thermal injury: II. The relative importance of time and surface temperature in the causation of cutaneous burns. Am J Pathol 23: 695-720.

12. American Thoracic Society/European Respiratory Society (2002) ATS/ERS statement on respiratory muscle testing. Am J Respir Crit Care Med 166: 518624.

13. Spielberger CD, Gorsuch RL, Lushene RE (1970) STAI manual. Palo Alto, Consulting Psychologist Press. 